\title{
Téoros
}

Revue de recherche en tourisme

\section{Transport aérien et tourisme régional : le cas des Iles de la Madeleine}

\section{Claude Richard}

Volume 6, numéro 1, mars 1987

Tourisme et transports

URI : https://id.erudit.org/iderudit/1080520ar

DOI : https://doi.org/10.7202/1080520ar

Aller au sommaire du numéro

Éditeur(s)

Université du Québec à Montréal

ISSN

0712-8657 (imprimé)

1923-2705 (numérique)

Découvrir la revue

Citer cet article

Richard, C. (1987). Transport aérien et tourisme régional : le cas des Iles de la

Madeleine. Téoros, 6(1), 11-12. https://doi.org/10.7202/1080520ar d'utilisation que vous pouvez consulter en ligne.

https://apropos.erudit.org/fr/usagers/politique-dutilisation/ 


\title{
Transport aérien et tourisme régional: Le cas des lles de la Madeleine
}

\author{
par Claude Richard*
}

La notion même de tourisme inclut par définition celle de déplacement, donc de transport. Le transport aérien est sans contredit le vêhicule qui a le plus largement contribué a l'expansion du tourisme mondial au cours de la dernière décennie. Choisir les Iles de la Madeleine comme exemple pour illustrer les incidences du transport aérien sur le développement touristique régional, c'est probablement choisir la destination québécoise où ces interdépendances se présentent avec le plus d'évidence. C'est aussi parler d'un lieu où les questions de transport, et notamment de transport aérien, sont intimement liées aux préoccupations les plus quotidiennes des gens qui y habitent, ceci allant de l'achat du journal quotidien qui arrive par avion depuis Montréal ou Québec jusqu'à l'importation de presque toutes les denrées alimentaires à une certaine période de l'année.

\section{Le tourisme: un développement récent}

Le court historique du tourisme aux lles de la Madeleine peut, quant à lui, s'inscrire en parallèle avec celui des plus récents développements en transports et communications. Ainsi, il a vraiment commencé à être question de tourisme aux Iles à partir de 1971 avec la mise en service du premier traversier depuis l'Tle du Prince-Edouard. Dans les années qui ont suivi, les capacités d'accueil de ce transporteur, auxquelles venaient s'ajouter celles de deux vols quotidiens, sont venues en quelque sorte fixer les limites d'entrée aux lles et ont pour ainsi dire trace la configuration du développement touristique des années ' 70 et du début des années ' 80 .

Toute une infrastructure locale de support et d'accueil s'est graduellement développée pour répondre aux besoins de cette nouvelle demande croissante jusqu'en 1980 puis restée relativement stable au cours des derritères années sauf pour une récente remontée depuis 1985. C'est donc un peu plus d'une vingtaine de mille visiteurs, pour la grande majorité de provenance québecoise, qui se rendent aux lles principalement en juillet et aoutt pour un scjour moyen d'une

"M. Claude Richard est directeur de l'Association touristique des llas de la Madeleine.

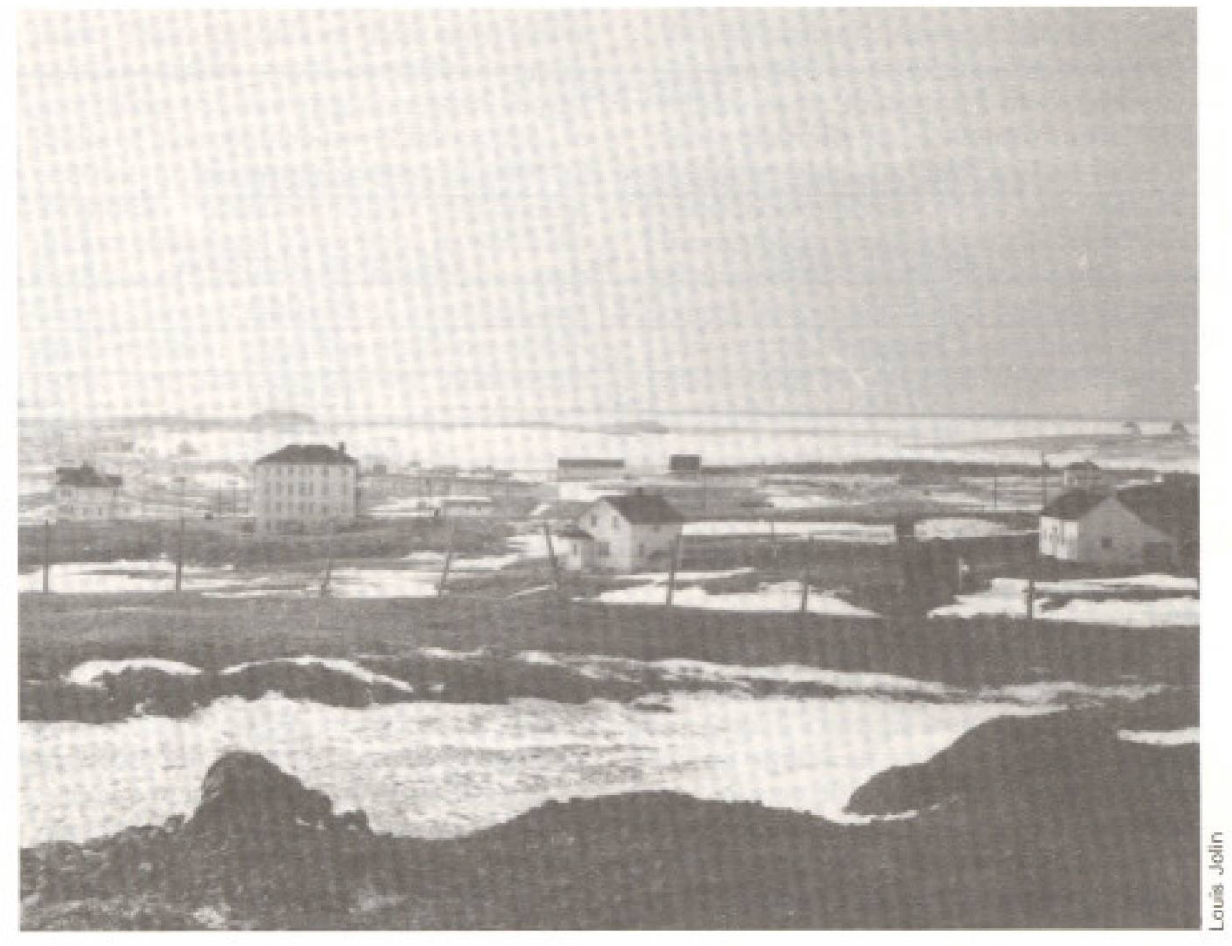

semaine. Aprè̀s une quinzaine d'années d'expérience touristique, ce phénomène fait maintenant vraiment partic du portrait socio-b́conomique de la région et c'est dans une nouvelle perspective que se pose maintenant le développement du tourisme: consolidation du produit, étalement de la saison, développement de sous-produits et de nouveaux marchés, marketing...

\section{La situation}

Avec une population de quelque 14000 habitants et une affluence touristique qui se chiffrait à tout près de 24000 visiteurs au cours de l'été 86 , les Iles de la Madeleine sont actuellement desservies par deux compagnies aériennes qui les relient chacune au continent sur une base quotidienne et une compagnie locale de transport maritime qui opère un service de traversier entre les lles et l'Ile du Prince-Edouard ainsi qu'un service de cargo entre les lles et Montréal de neuf à dix mois par année tout dépendant des conditions de navigation qui prévalent dans le golfe Saint-Laurent de décembre à avril.
Le transport aérien aux lles de la Madeleine doit done composer avec, d'une part, les besoins d'une population résidente sur une base annuelle, pour qui il constitue un service essentiel et, d'autre part, une demande touristique concentrée presqu'exclusivement sur les mois de juillet et aoutt. Les besoins de ces deux clientèles distinctes peuvent diverger en matière d'itinéraires et de fréquences de vols et on a souvent dû se rendre a l'kvidence que toute intervention sur l'un ou l'autre aspect du service aérien dans le but d'avantager une clientèle peut présenter des inconvénients pour l'autre partie concernée.

Contrairement à la plupart des destinations touristiques étrangères où le transport aćrien vers le tourisme reste bien dissocie du transport qui dessert la population résidente, l'organisation du tourisme à forfaits a toujours êté jusqu'ici intégrée aux réseaux de transport régional. On n'a donc pas en direction des lles de vols "charters" independants des vols réguliers qui desservent la destination sur une base regulière. Autre fait particulier en milieu insulaire, on ne 
STATISTIQUES-PASSAGERS TABLEAU 1

(1981-1986)

\begin{tabular}{|c|c|c|c|c|}
\hline \multicolumn{5}{|c|}{ TABLEAU 1 } \\
\hline & $\begin{array}{c}\text { Transport aérien } \\
(12 \text { mois) }\end{array}$ & $\%$ & $\begin{array}{c}\text { Transport maritime } \\
(-10 \text { mois) }\end{array}$ & $\%$ \\
\hline 1986 & 19427 & 43 & 25984 & 57 \\
\hline 1985 & 18850 & 45 & 22727 & 55 \\
\hline 1984 & 18400 & 48 & 20244 & 52 \\
\hline 1983 & 15100 & 41 & 21754 & 59 \\
\hline 1982 & 16900 & 43 & 22763 & 57 \\
\hline 1981 & 19900 & 45 & 24297 & 55 \\
\hline
\end{tabular}

\section{TABLEAU 2}

\begin{tabular}{|c|c|r|c|r|}
\hline \multicolumn{7}{|c|}{ Entrées-passagers aux lles: du $1^{\text {er juin - } 15 \text { septembre }}$} \\
\hline & Transport aérien & $\%$ & Transport maritime & $\%$ \\
\hline 1986 & 7633 & 27 & 21043 & 73 \\
\hline 1985 & 7485 & 30 & 17830 & 70 \\
\hline 1984 & 6707 & 30 & 15527 & 70 \\
\hline 1983 & 5890 & 26 & 16795 & 74 \\
\hline 1982 & 6665 & 27 & 17819 & 73 \\
\hline 1981 & 7280 & 27 & 19533 & 73 \\
\hline
\end{tabular}

peut envisager la question du transport aérien sans considérer celle du transport maritime, seule autre voie d'accès aux Iles. En se reportant aux tableaux 1 et 2 , on pourra observer en meme temps qu'une courbe de l'évolution du trafic vers les Iles, dans quelle proportion les voyageurs ont choisi l'un ou l'autre de ces deux modes de transport, ce sur une base annuelle et en saison touristique plus particulièrement.

L'écart observé au tableau 2 entre le trafic aérien et le trafic maritime en saison estivale s'explique par le fait que pres de $80 \%$ de la clientele touristique se rend aux Iles en empruntant la route et le traversier à partir de l'lle du Prince-Edouard. En dehors, de la période estivale tout particulièrement, la population résidente utilise davantage l'avion que le traversier pour aller à l'extérieur; ce qui, à l'échelle annuelle, réduit considérablement l'ecart entre le trafic maritime et le trafic aérien. Entre 1983 et 1984, la marge qui sépare ces deux modes de transport est passée de quelque 7000 à moins de 2000 passagers. Il existe donc une demande potentielle importante pour le transport aérien mais ce sont les conditions offertes au voyageur (tarifs, itinćraires, types d'avions...) qui vont influencer directement son taux d'utilisation.

On choisira davantage le transport aćrien pour sa rapidité et son confort mais à condition toutefois que les conditions proposees demeurent incitatives. C"est ainsi qu'à l'êté 84 , suite à une campagne de promo- tion bien médiatisée de la compagnie Québécair accompagnée de tarifs excursions très avantageux, on a vu le nombre de passagers sur cette compagnie augmenter de quelque $40 \%$ par rapport à l'été précédent et le trafic maritime diminuer de quelques milliers de passagers pour la même période.

\section{Perspectives de développement}

Il faut par ailleurs considérer la situation de concurrence dans laquelle est placée une destination comme les Iles de la Madeleine. Leur position géographique implique en effet des coûts d'accès comparables à des destinations internationales qui héritent d'une plus longue tradition touristique et bénéficient par conséquent de moyens promotionnels beaucoup plus importants; donc, concurrence avec des destinations tout aussi "exotiques" et souvent plus facilement accessibles. Par ailleurs, les marchés actuels et potentiels ne se situant pas chez nos voisins immédiats, les Maritimes par exemple, mais à des distances appréciables (Montréal et Québec à plus de 1000 kilomètres et bien davantage encore, si on pense aux marchés hors-Québec), il devient impératif de nous "rapprocher" de ces clientèles, ce qui se traduit par une nécessaire évolution du transport aérien qui dessert les Iles.

Quant à la plupart des prévisions en matière de développement touristique, elles passent obligatoirement par la mise en marche de forfaits, qu'il s'agisse d'étalement de la saat- son en fonction de clientèles spécifiques, de thèmes particuliers (saison du homard, observation des phoques, activités nautiques...) ou encore simplement de rendre la destination mieux accessible sur des nouveaux marchés.

Cependant la mise en marché de forfaits touristiques concurrentiels rencontre certaines contraintes comme l'indexation aux coûts déjà très élevés du transport régional au Québec et le besoin d'une meilleure adequation des structures aćroportuaires ou de certains éléments de support comme l'hébergement et les services touristiques qui s"y rattachent. C'est ainsi que depuis plusicurs annés, une partie importante des intervenants régionaux concemés croit que l'expansion du tourisme à forfaits implique nécessairement une modification des infrastructures aéroportuaires qui permettrait l'atterrissage d'avion de type gros porteurs, entraînant ainsi une croissance graduelle du nombre d'unites d'hébergement et des services touristiques connexes, pour aussi faire tourner la roue sur une plus grande echelle.

Toutefois, certaines divergences se manifestent à ce sujet à l'intérieur mème des instances locales et gouvernementales impliquées en même temps que les différentes conséquences de la crise énergétique et économique du début des années ' 80 ont jusqu'ici ralenti ces nouveaux développements. Or, le tout récent contexte de la déréglementation et de la privatisation dans le monde du tansport aćrien va maintenant foumir une nouvelle toile de fond à ce débat qu'on s'attend à revoir apparaítre incessamment.

En conclusion, il nous apparaît à peu près évident que, tout comme l"amélioration de la liaison maritime par le traversier a servi de "déclencheur" "à l"industrie touristique aux lles, le second souffle du développement passe par une évolution du transport aérien qui relie les Iles avee le continent et avec Montréal d'abord, permettant une meilleure possibilité de connexions avec des marchés potentiels. C'est donc dire que l'avenir du tourisme régional reste en partie tributaire de la situation générale du transport aérien au Québec, elle-même déja orientée par des décisions prises à des niveaux qui semblent vouloir echapper de plus en plus au contrồle des intervenants régionaux.

C'est sous forme d'interrogation que nous nous concluerons ce bref expose d'un cas qui, tout particulier qu'il soit, n'est pas sans présenter certaines ressemblances avec d'autres régions "éloignées" des grands centres. En effet, la grande question reste de savoir dans quelle mesure le récent contexte de la déréglementation qui déterminera dans les années à venir les règles du jeu dans le monde du transport aérien saurat-il favoriser l'accès des destinations horscentres aux grands réseaux qui sont ceux du tourisme mondial... $f$ 